Xuewei Jiang*, Yu Chen, Yue Yuan, and Lu Zheng

\title{
Thermal Response in Cellulose I $\beta$ Based on Molecular Dynamics
}

https://doi.org/10.1515/cmb-2019-0007

Received September 8, 2019; accepted November 21, 2019

\begin{abstract}
The structural details of cellulose I $\beta$ were discussed according to molecular dynamics simulations with the GLYCAM-06 force field. The simulation outcomes were in agreement with previous experimental data, including structural parameters and hydrogen bond pattern at $298 \mathrm{~K}$. We found a new conformation of cellulose $\mathrm{I} \beta$ existed at the intermediate temperature that is between the low and high temperatures. Partial chain rotations along the backbone direction were found and conformations of hydroxymethyl groups that alternated from $t g$ to either $g t$ or $g g$ were observed when the temperature increased from $298 \mathrm{~K}$ to $400 \mathrm{~K}$. In addition, the gg conformation is preferred than gt. For the structure adopted at high temperature of $500 \mathrm{~K}$, major chains were twisted and two chains detached from each plain. In contrast to the observation under intermediate temperature, the population of hydroxymethyl groups in gt exceeded that in gg conformation at high temperature. In addition, three patterns of hydrogen bonding were identified at low, intermediate and high temperatures in the simulations. The provided structural information indicated the transitions occurred around $350 \mathrm{~K}$ and $450 \mathrm{~K}$, considered as the transitional temperatures of cellulose $\mathrm{I} \beta$ in this work.
\end{abstract}

Keywords: Thermal response, Molecular dynamics, Structural properties, Hydrogen bonding pattern, Transition temperature

MSC: $92 \mathrm{C} 40$

\section{Introduction}

The research tendency in cellulose and its applications has increased extensively during the past few years (Bergenstrahle et al. 2007; Chen et al. 2018; Miyamoto et al. 2016; Paavilainen et al. 2011; Zhang et al. 2011). As an important natural polymer to industrialization, cellulose can be extracted from various plants and has been widely used to produce materials such as textiles, pulp, biofuels, etc. (Liu et al. 2011; Mazeau 2005; Wyman 2007). In addition, cellulosic fiber and regenerated cellulosic fiber are principal raw materials for textile industry and medical application (Mazeau 2005). Therefore, research in the effect of temperature on the structure and mechanical properties of the cellulose crystal can be significant not only to understanding the fundamental mechanisms of the complex cellulosic self-assembly formation, but also for redesigning the sustainable regenerated cellulosic materials with desired performance (Bergenstrahle et al. 2007; Zhang et al. 2011).

Structure wise, cellulose is linear-chain polymer that consists of D-glucose repeat units. The hydroxyl groups between adjacent chains contribute to the hydrogen bond patterns in crystalline structure. Divergent morphologies of cellulose crystals were adopted in nature (Atalla and Vanderhart 1984), and cellulose with crystalline form I $\beta$ constitutes the secondary wall of cotton fiber, which is one of the most abundant nature

\footnotetext{
*Corresponding Author: Xuewei Jiang: Wuhan Textile and Apparel Digital Engineering Technology Research Center, Wuhan Textile University, Wuhan 430073, Hubei, China

Hubei Key Laboratory of Biomass Fibers and Eco-dyeing \& Finishing, Wuhan Textile University, Wuhan 430073, China, E-mail: xwjiang@wtu.edu.cn

Yu Chen, Lu Zheng: Wuhan Textile and Apparel Digital Engineering Technology Research Center, Wuhan Textile University, Wuhan 430073, Hubei, China

Yue Yuan: Department of Textile Engineering, Chemistry and Science, North Carolina State University, USA 27067
} 
fibers on earth (Horii et al. 1987; Sugiyama et al. 1991). Cellulose crystal in I $\beta$ form has a typical structure in which cellulosic chains are arranged to form parallel sheets (Gardner and Blackwell 1974). The sheets are stabilized by hydrogen bonds while the stacking of the sheets are stabilized by both Van der Waals forces and weak intersheet hydrogen bond interactions (Nishiyama et al. 2008; Nishiyama et al. 2002). In a polymersolvent system, the hydrogen bonds are critical to the dissolution and stability of the polymer molecules (Cai et al. 2012; Chen et al. 2011; Makhatadze and Privalov 1992; Pace 1986; Swatloski et al. 2002; Zou et al. 2002). Therefore, the computational and experimental works on cellulose currently concentrated on the analysis of the hydrogen bonds in order to study the mechanical properties of cellulosic materials (Cai et al. 2012; Hayakawa et al. 2017; Heiner et al. 1998; Heiner et al. 1995; Miyamoto et al. 2013; Rabideau et al. 2013; Shiiba et al. 2013; Yamane et al. 2013; Yui et al. 2006). To investigate the change of structural and mechanical properties of cellulose in responding to the temperature, parameters such as the density, torsion angle, crystal unit cell parameters, hydrogen bond pattern etc. have been used as indicators in previous studies of cellulose thermal behavior.(Bergenstrahle et al. 2007; Nishiyama et al. 2002; Nishiyama et al. 2003; Zhang et al. 2011)

Among all the physical properties of cellulose, thermal behavior is one of the most critical properties for the material processing and research has been done in studying cellulose thermal stability with a temperature swing (Zhang et al. 2011). Cellulosic materials were subjected to dynamic thermogravimetry analysis in both nitrogen and air by Huang et al. (Huang and Li 1998), giving that an approximated range of initial degradation temperatures of cellulose is between 546 and $612 \mathrm{~K}$. The measured values of initial thermal degradation temperature and the temperature where the maximum degradation rate occurs were used to describe the thermal stability of the cellulose and its esters. The thermal behavior of cellulose $\mathrm{I} \beta$ has been studied experimentally by Wada et al. (Wada 2002; Wada et al. 2010) and Watanabe et al. (Watanabe et al. 2006a; Watanabe et al. 2006b) using X-ray diffraction and temperature-dependent infrared spectroscopy (IR) measurements. According to the temperature dependent IR measurements, thermolysis of cellulose in $\mathrm{I} \beta$ crystalline form has not been detected at the temperature from $303 \mathrm{~K}$ to $533 \mathrm{~K}$, although significant changes of hydrogen bonds were observed (Watanabe et al. 2006a). These divergent experimental results suggest a necessary investigation of the thermal response of cellulose I $\beta$ crystals, the existence of transition temperature, the temperature-dependent hydrogen bond formation and the hydrogen bond patterns.

However, experimental studies have limitations in tracking the thermal response of cellulose structure along a temperature change at molecular level. Instead, molecular dynamics (MD) simulations can act as powerful tools in monitoring the structural alternation of cellulose under heating. The previous works have studied the flexibility and interaction strength of cellulose molecules at ambient conditions using MD simulations (Nishiyama et al. 2008; Zhang et al. 2011). In MD simulation, the accuracy of outcomes is highly relied on the selection of force field. Stortz and his co-workers (Stortz et al. 2009) compared empirical force fields and the semi-empirical quantum methods when studying carbohydrates, such as $\beta$-cellobiose, $\alpha$-maltose, and $\alpha$-galabiose. They concluded that the force fields GLYCAM-06, GROMOS, and MM3 are preferred with above carbohydrate molecules. In addition, the GLYCAM-06 force field is consistent and transferable for modeling carbohydrates (Kirschner et al. 2008), lipids (Tessier et al. 2008), and glycoconjugates (DeMarco and Woods 2009; DeMarco et al.2010). Spiwok et al. (Spiwok et al.2010) found that the results of the surface of free energy of $\beta$-D-glucopyranose using that force field were agree with the results of quantum metadynamics studies, providing a higher accuracy of the simulation process.

Here in the present work, we applied the GLYCAM-06 force field to study the thermal behavior of cellulose $\mathrm{I} \beta$ with a temperature swing from $298 \mathrm{~K}$ to $550 \mathrm{~K}$. We carried out MD simulations with 36 cellulose chains under the NPT ensemble. Beyond the room temperature phase and high temperature phase of cellulose I $\beta$ that were discussed in literature (Wada et al. 2010), our simulation results suggested the existence of an intermediate structure of cellulose I $\beta$. At this intermediate state, the hydroxymethyl groups adopt either $\mathbf{g t}$ or $\mathbf{g g}$ conformation and twisting was observed within some polymer chains. To fully understand the thermal behavior of cellulose, further analysis of the structure-thermal property correlation of cellulose I $\beta$ was carried out. 


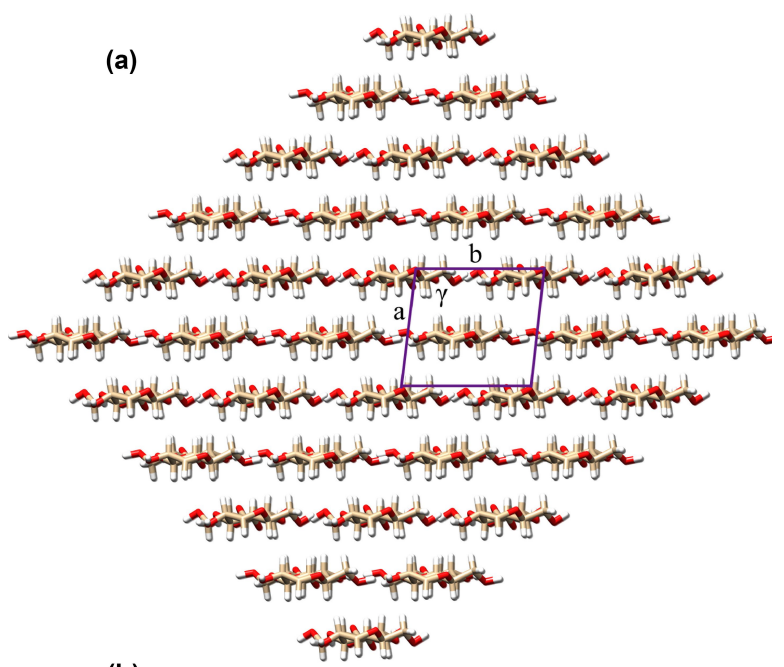

(b)

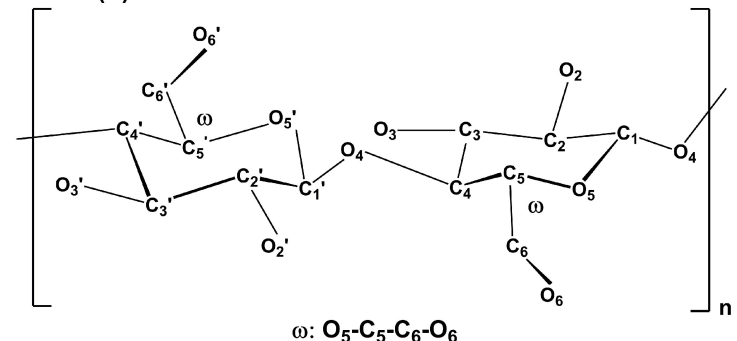

Figure 1: (a) The model of cellulose projected onto the ab base plane. The model was constituted by $6 \times 6$ chains, each consisting of eight glucose units. (b) Schematic representation of the cellobiose repeat unit with definitions of conformational torsion angles.

\section{Methods}

\subsection{Initial structure and conformational parameters}

Cellulose elementary fibril with 36 cellulose chains (Fig. 1a) proposed by Ding and Himmel (Ding and Himmel 2006) was used to build the model of cellulose $I \beta$ here. The raw atomic coordinates and the positions of hydroxyl groups are assigned according to the crystallographic unit cell data and dominant hydrogen bonding pattern, which was determined by X-ray and neutron fiber diffraction refinement experimental methods (Nishiyama et al. 2002). The initial structure was generated by a user-friendly program named cellulosebuilder (Gomes and Skaf 2012). The unit cell belongs to the monoclinic space group $P 2{ }_{1}$ and conformational parameters are $\boldsymbol{a}=7.784 \AA, \boldsymbol{b}=8.201 \AA, \boldsymbol{c}=10.38 \AA$ and $\gamma=96.5^{\circ}$, where $\boldsymbol{c}$ is the chain direction and unique axis. Cellulose chains are packed parallel and each chain contains eight glucoses units.

The conformational parameters of the cellulose chains are shown in Fig. 1(b). The unit cell $\boldsymbol{a}, \boldsymbol{b}, \boldsymbol{c}, \alpha, \beta$, and $\gamma$ are commonly used to describe the crystal structure. The $\boldsymbol{a b}$ projection of one of considered unit cells is shown in Fig. 1a. The unit cell $c$ represents the length of one cellobiose unit alone chain direction. The unit $\alpha$ is the angle between directions of $\boldsymbol{b}$ and $\boldsymbol{c}$, whereas $\beta$ is the angle between directions of $\boldsymbol{a}$ and $\boldsymbol{c}$. Thermal characters are evaluated according to thermal variations of these unit cell parameters. Torsion angle 05 - 5 C6-O6 at the $\mathrm{C} 6$ primary alcohol group is defined as $\omega$ (Fig. 1b). The dihedral angle $\omega$ has three staggered positions at $60^{\circ}, 180^{\circ}$, and $300^{\circ}$ and the conformations of the hydroxymethyl groups are labeled $\mathbf{g t}$, $\mathbf{t g}$ and gg respectively. 


\subsection{System optimization}

The solvent box was set as a cube with a given distance of 12 A and the TIP3P water model was used (Jorgensen et al. 1983). Non-bonded interactions were calculated with a cutoff radius of 12 Å. GLYCAM-06 force field was applied in this study and it has been extensively used in simulations of carbohydrate (Kirschner et al. 2008). Some previous works also shown that the force field GLYCAM-06 was suitable for the MD simulation at high temperature (Bergenstrahle et al. 2007; Zhang et al. 2011).

The energy minimization procedure consists of two stages, first for the solvation only then for the whole system. In the energy minimization of solvation, 2000 steps of steepest descent minimization were performed followed by 3000 steps of conjugate gradient minimization. Prior to the minimization of energy of the solvated system, cellulose molecules were fixed with positional restraints. To fully optimize the structure, the energy of entire system is minimized with 2000 steps of steepest descent minimization and then 3000 steps of conjugate gradient minimization.

\subsection{Molecular dynamics simulation}

To investigate the thermal response of cellulose in crystalline I $\beta$ form, we carried out MD simulations at different temperatures ( $298 \mathrm{~K}, 350 \mathrm{~K}, 400 \mathrm{~K}, 450 \mathrm{~K}, 500 \mathrm{~K}$ and $550 \mathrm{~K})$. All simulations were conducted by using Amber software (D.A. Case et al. 2012). The equilibration process was performed prior to the production therefore the system can be heated up from $0 \mathrm{~K}$ to the target temperature. In order to avoid wild fluctuations and hot solvent cold solute etc., the Langevin temperature equilibration scheme was used for the equilibrium of the system temperature (Sindhikara et al. 2009). Due to the inaccuracy of the pressure calculation at first few picoseconds (ps) under low temperatures, one nanosecond (ns) of simulations at the NVT ensemble with restraints were performed initially for all conditions. All bonding involved hydrogen atoms were constrained by the SHAKE algorithm simultaneously (Miyamoto and Kollman 1992). Then following simulations were performed under NPT ensemble with the pre-determined temperature. The Berendsen barostat was used to maintain the pressure around $1 \mathrm{~atm}$ and the relaxation time was set as 2 ps (Berendsen et al. 1984). Same to the equilibrium steps, SHAKE algorithm was applied to constrain binding involved hydrogen atoms and the Langevin dynamics was used for the temperature controlling (Miyamoto and Kollman 1992; Sindhikara et al. 2009). The time step was set as 2 femtoseconds (fs) and the snapshots were saved every $10 \mathrm{ps}$ at desired temperature. $100 \mathrm{~ns}$ MD simulations were performed at $298 \mathrm{~K}, 350 \mathrm{~K}, 400 \mathrm{~K}$ and $450 \mathrm{~K}$. In order to ensure the equilibrium was achieved with sufficient time period, $200 \mathrm{~ns}$ simulations were run for $500 \mathrm{~K}$ and $550 \mathrm{~K}$.

Additional 10 sets of $5 \mathrm{~ns}$ simulations with different starting configurations based on 100 ns simulation trajectories were done under each temperature to enhance the validity of our simulations. The random number generators were used in each MD simulation. From these MD simulation sets, errors can be estimated for different parameters (density, unit cell, hydrogen bonding and torsion angle etc.) calculated in simulations and the average values were used for further analysis.

\section{Results and Discussions}

\subsection{Equilibration of system}

The root means square deviation (RMSD) of the atom C1, C2, C3, C4, C5 and 05 and total energy were used to evident the equilibration of system at different temperatures. A stable RMSD and total energy value indicates the equilibration was achieved in the system. Different time lengths were required to achieve stable values under different temperatures (Fig. 2).

The curve of simulation at $298 \mathrm{~K}$ in Fig. 2(a) demonstrates that the mean of RMSD is around $0.49 \AA$ and the total energy is around $-40000 \mathrm{kcal} / \mathrm{mol}$ (Fig. 2(b)). The system reached equilibrium immediately, indicating that the backbone structure changes negligibly to initial structure at the room temperature. The mean 

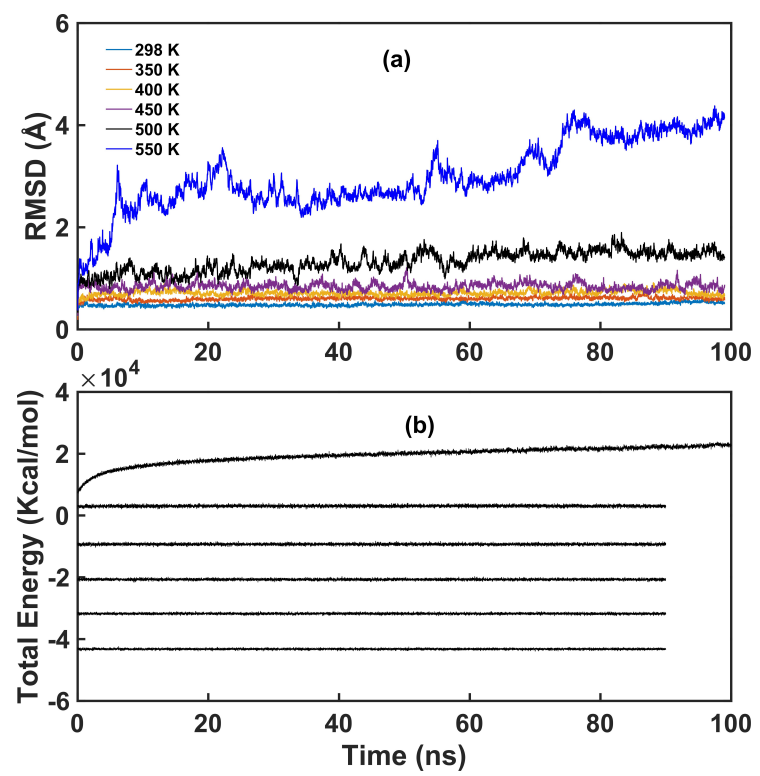

Figure 2: (a) RMSD for cellulose $\mathrm{I} \beta$ at temperature from $298 \mathrm{~K}$ to $550 \mathrm{~K}$ respectively. (b) Total energy for cellulose $\mathrm{I} \beta$ at temperature from $298 \mathrm{~K}$ to $550 \mathrm{~K}$ respectively.

values of RMSD are about $0.60 \AA$, $0.72 \AA$ and $0.85 \AA$ at $350 \mathrm{~K}, 400 \mathrm{~K}$ and $450 \mathrm{~K}$ respectively. The mean values of total energy are about $-32000 \mathrm{kcal} / \mathrm{mol},-20100 \mathrm{kcal} / \mathrm{mol}$ and $-9100 \mathrm{kcal} / \mathrm{mol}$ at $350 \mathrm{~K}, 400 \mathrm{~K}$ and $450 \mathrm{~K}$ respectively. These results are consistent with the results reported by Zhang et al. that the system reaches equilibrium immediately and the change of backbone structure can be ignored under aforementioned temperatures (Zhang et al. 2011). The mean of RMSD is nearly $1.46 \AA$ at temperature $500 \mathrm{~K}$ and the total energy is nearly $50 \mathrm{kcal} / \mathrm{mol}$. This indicates that the system achieves equilibrium after $50 \mathrm{~ns}$ (Fig.2, a). According to the nonlinear increasing of RMSD from $450 \mathrm{~K}$ to $500 \mathrm{~K}$, an alteration of cellulose I $\beta$ molecular structure occurred under higher temperature. At $550 \mathrm{~K}$, the system reached equilibrium after $75 \mathrm{~ns}$ with a mean of RMSD about $3.72 \AA$ and the total energy about $20000 \mathrm{kcal} / \mathrm{mol}$. This further increased RMSD indicates that the molecular structure of cellulose I $\beta$ experienced significant changes under this temperature with a slow pace in achieving equilibrium. In general, the cellulose I $\beta$ system reached a temporary equilibrium in first $20 \mathrm{~ns}$ regardless the simulation temperature, which is consistent with the previous study (Zhang et al. 2011). However, the structure of system changes significantly within first $75 \mathrm{~ns}$ at high temperature.

\subsection{Structural properties}

The density, unit cell parameters and torsion angles were used to represent the structural proprieties of cellulose $\mathrm{I} \beta$ at different temperatures in the simulation. The MD simulation trajectories were utilized to investigate influence of temperature on the structure and correlated properties from $298 \mathrm{~K}$ to $550 \mathrm{~K}$.

The crystal density is a macroscopic parameter of cellulose in $\mathrm{I} \beta$ crystalline form and is a critical indicator of its structural characters. The density is convertible so that the constant pressure is retained while performing MD simulation in NPT ensemble. The average density evolution with temperature was shown in Fig. 3. At the room temperature (298K), the average equilibrated density was found about $1587 \mathrm{~kg} / \mathrm{m}^{3}$ with a standard deviation of $2.92 \mathrm{~kg} / \mathrm{m}^{3}$. This value is less than the experimental density of $1636 \mathrm{~kg} / \mathrm{m}^{3}$ estimated from the crystal cell parameters (Nishiyama et al. 2002). The difference between the density obtained from simulation and the experimental value is about $3.0 \%$. In addition to evaluate the accuracy of our simulation by comparing with experimental data at $298 \mathrm{~K}$, we monitored the density change of cellulose $\mathrm{I} \beta$ when raising of the temperature. The general trend shows that the density of cellulose molecules decreased when increasing the temperature. The density decreases about 3.4\% as the temperature changes from $298 \mathrm{~K}$ to $350 \mathrm{~K}$. This 


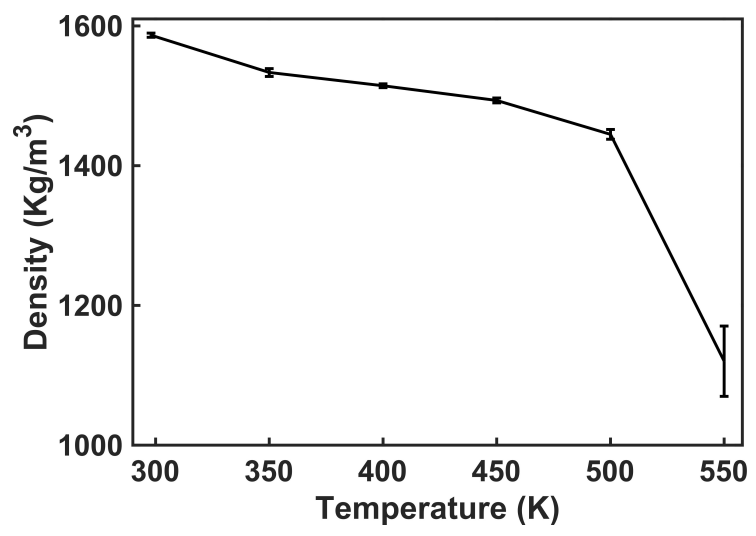

Figure 3: Average densities as a function of temperature from $298 \mathrm{~K}$ to $550 \mathrm{~K}$.

is greater than that when the temperature raised from $350 \mathrm{~K}$ to $450 \mathrm{~K}$, which is about $1.3 \%$. However, the density decreases nonlinearly by about $3.2 \%$ as the temperature increases to $500 \mathrm{~K}$ from $450 \mathrm{~K}$ and a sudden drop occurs by about $22.5 \%$ after heating the system to $550 \mathrm{~K}$. The sudden drop of density of cellulose was reported at 453K-523K in experimental studies for various samples (Swatloski et al. 2002; Zou et al. 2002) and a range of 450-500K was reported in MD simulation of cellulose I $\beta$ (Wyman 2007). According to that reported in previous studies by both experiments and molecular dynamics simulations, a temperature where a sudden drop of cellulose density happened is considered as the transition temperature (Swatloski et al. 2002; Wyman 2007; Zou et al.2002). At this temperature, the structural properties of cellulose have significant changes. Our simulation results (Fig. 3) shown a similar trend when comparing with these literatures, indicating that the structure of cellulose is becoming slightly looser with the temperature increasing and discussion ensues.

Besides the density estimation above, the unit cell parameters of each glucose units were calculated in this work under various temperatures. Unit cell parameters were measured by locally regarding 60 different unit cells for different temperature. Corresponding distances and angles between 04 atoms were traced during the last 4 ns temperature (Bergenstrahle et al. 2007; Zhang et al. 2011). The unit cell parameters obtained from simulation are listed in Table 1 along with the experimental and simulation results from literature (Bergenstrahle et al. 2007; Nishiyama et al. 2002; Wada et al. 2010; Zhang et al. 2011). The unit cell parameters $\boldsymbol{a}, \boldsymbol{b}$ and $\boldsymbol{c}$ at $298 \mathrm{~K}$ are $7.78 \AA$, $8.23 \AA$ and $10.78 \AA$ respectively. The largest deviation in length occurs in unit cell parameter $\boldsymbol{c}(3.9 \%)$, while the deviations for other unit cell lengths are less than $0.5 \%$ compared to the experimental results (Nishiyama et al. 2002). The predicted unit cell angles $(\alpha, \beta$ and $\gamma$ ) are 89.30, 91.25 and $98.23^{\circ}$ respectively, which match perfectly with the experimental values (Nishiyama et al. 2002). The predicted values deviate from the experimental results by only $1.7 \%$. All unit cell parameters are within the range of reported experimental values. These results indicate that the initial structure and hydrogen bonds in cellulose $\mathrm{I} \beta$ are properly revealed by our model. According to the agreements between values obtained from our simulations and results from experiment or other simulations, we confirmed that GLYCAM-06 that we chose to predict unit cell parameters are valid and suitable in studying the thermal response of cellulose I $\beta$ molecules (Nishiyama et al. 2002; Wada et al. 2010).

The structure alternation within temperature range is unveiled by the variation of the average values for all unit cell parameters at different temperatures as shown in Fig. 4. Significant increases of unit cell $\boldsymbol{a}, \boldsymbol{b}$ and $\beta$ were observed when temperature raised from $298 \mathrm{~K}$ to $500 \mathrm{~K}$. Therefore, structure at $500 \mathrm{~K}$ can be deemed as a representative of cellulose $\mathrm{I} \beta$ structure at a high temperature. In addition, change of the unit cell angles $(\alpha, \beta$, and $\gamma)$ at high temperature are smaller than $0.7 \%$ when compared with that at $298 \mathrm{~K}$.

The unit cell parameter $\boldsymbol{a}$ is $8.1 \%$ longer than that at the low temperature, which is comparable with the 5.3\% detected by experiment (Wada 2002) and 7.5\% by another MD simulation (Bergenstrahle et al. 2007). The parameter $\boldsymbol{b}$ increases merely $1.8 \%$ from $298 \mathrm{~K}$ to $500 \mathrm{~K}$ in our simulation, but it was reported with an about $6 \%$ increase from 298K in previous work (Bergenstrahle et al. 2007). At $500 \mathrm{~K}$, unit cell $\boldsymbol{c}$ increases infinitesimally, which is consistent with other works (Bergenstrahle et al. 2007; Zhang et al. 2011). Although 
Table 1: Average Unit cell axial length $(\AA)$ and angles $(\stackrel{\circ}{)}$

\begin{tabular}{|c|c|c|c|c|c|c|}
\hline \multicolumn{3}{|c|}{ Unit cell axial length $(\AA ̊)$} & \multicolumn{3}{|c|}{ Unit cell axial angles $\left(^{\circ}\right)$} & \multirow[t]{2}{*}{ reference } \\
\hline $\mathrm{a}$ & $\mathrm{b}$ & c & $\alpha$ & $\beta$ & $\gamma$ & \\
\hline 8.38 & 8.17 & 10.52 & 89.97 & 89.97 & 90.9 & MD $300 \mathrm{~K}$ (Bergenstrahle et al. 2007) \\
\hline 9.01 & 8.66 & 10.47 & $77.62 / 103.2$ & $78.30 / 101.4$ & 88.3 & MD $500 \mathrm{~K}$ (Bergenstrahle et al. 2007) \\
\hline 7.63 & 8.23 & 10.80 & 89.99 & 89.99 & 97.2 & MD 298 K (Zhang et al. 2011) \\
\hline 8.11 & 8.28 & 10.78 & 90.00 & 89.96 & 98.3 & MD $500 \mathrm{~K}$ (Zhang et al. 2011) \\
\hline 7.78 & 8.20 & 10.38 & 90.00 & 90.00 & 96.5 & Experiment $300 \mathrm{~K}$ (Nishiyama et al. 2002) \\
\hline 8.19 & 8.18 & 10.37 & 90.00 & 90.00 & 96.4 & Experiment $523 \mathrm{~K}$ (Wada et al. 2010) \\
\hline 7.78 & 8.23 & 10.78 & 89.30 & 91.25 & 98.23 & This work $298 \mathrm{~K}$ \\
\hline 8.09 & 8.30 & 10.78 & 89.03 & 90.76 & 99.07 & This work $400 \mathrm{~K}$ \\
\hline 8.41 & 8.37 & 10.78 & 88.83 & 91.75 & 98.89 & This work $500 \mathrm{~K}$ \\
\hline
\end{tabular}
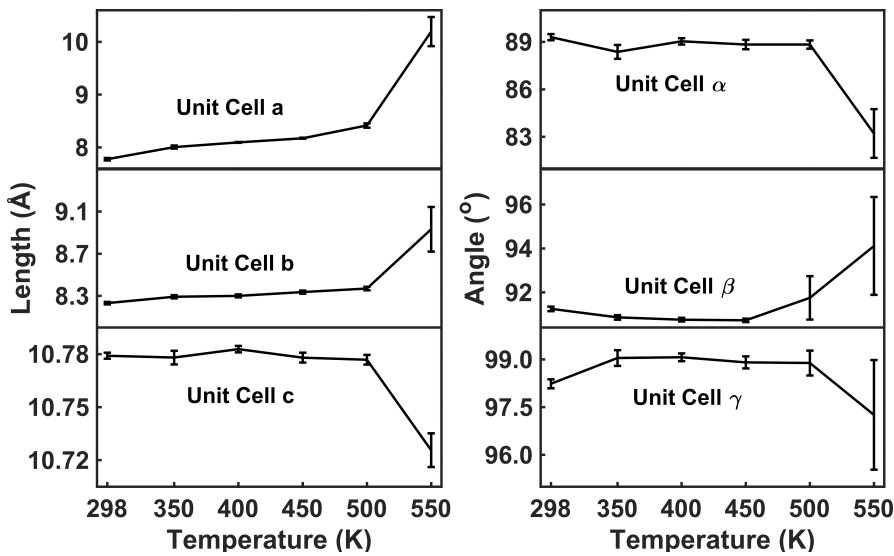

Figure 4: Variation of unit cell lengths $\boldsymbol{a}, \boldsymbol{b}$ and $\boldsymbol{c}$, angles $\alpha, \beta$, and $\gamma$ with temperature from $298 \mathrm{~K}$ to $550 \mathrm{~K}$.

the $\boldsymbol{b}$ axis in unit cell demonstrated difference in values, indeed anisotropic expansion thermal expansion was confirmed in our simulation results(Wada 2002). A sudden change in unit cell parameter is speculated on a transition in structure along the temperature change. Therefore, with the observation of the increased axis lengths $\boldsymbol{a}, \boldsymbol{b}$ and angle $\beta$, it can be concluded that the transition temperature of cellulose I $\beta$ lies in the temperature around $450 \mathrm{~K}$ at which the significant structural alternation occurred (Fig. 4).

An intermediate structure evidenced by the unit cell parameters was observed in between low and high temperature. The equilibrated structure at temperature $400 \mathrm{~K}$ was considered as the intermediate structure in our study and the structural parameters are listed in Table 1. The trend of unit cell parameters changes from $298 \mathrm{~K}$ to $550 \mathrm{~K}$ is plotted in Fig. 4. At $400 \mathrm{~K}$, the parameter $\boldsymbol{a}$ was increase about $4 \%$ while both parameters $\boldsymbol{b}$ and $\gamma$ were increased about $0.9 \%$, compared to that at $298 \mathrm{~K}$. When system was heated to $450 \mathrm{~K}$, only parameters $\boldsymbol{a}$ and $\boldsymbol{b}$ demonstrated a slight growth compared to that at $400 \mathrm{~K}$. Therefore, the changes of unit cell parameters from $298 \mathrm{~K}$ to $400 \mathrm{~K}$ are less than that from $400 \mathrm{~K}$ to $500 \mathrm{~K}$. In addition, a rearrangement of structure was observed from temperature $298 \mathrm{~K}$ to $400 \mathrm{~K}$ as shown in Fig. 5a and Fig. 5b. The minimal of unit cell parameter $\alpha$ was observed at $350 \mathrm{~K}$ (Fig. 4), suggesting that a temperature around $350 \mathrm{~K}$ can be considered as another transition temperature in addition to the $400 \mathrm{~K}$. Because the changes of the surface chains at the cellulose/water interface are caused by the water environment and are able to capture the fiber twist. This phenomena was considered as surface effects (Bergenstrahle et al. 2007; Yui et al. 2006). Therefore, the structure transition at $350 \mathrm{~K}$ is caused by the water environment and surface effects.

The graphical structures of the crystal structure equilibrated at temperature $298 \mathrm{~K}, 400 \mathrm{~K}$ and $500 \mathrm{~K}$ are shown in Fig. 5 as the representatives of structures at low (Fig. 5a), intermediate (Fig. 5b) and high temperature (Fig. 5c and Fig. 5d). It appears that the intermediate structure at $400 \mathrm{~K}$ tends to twist clockwise. The 

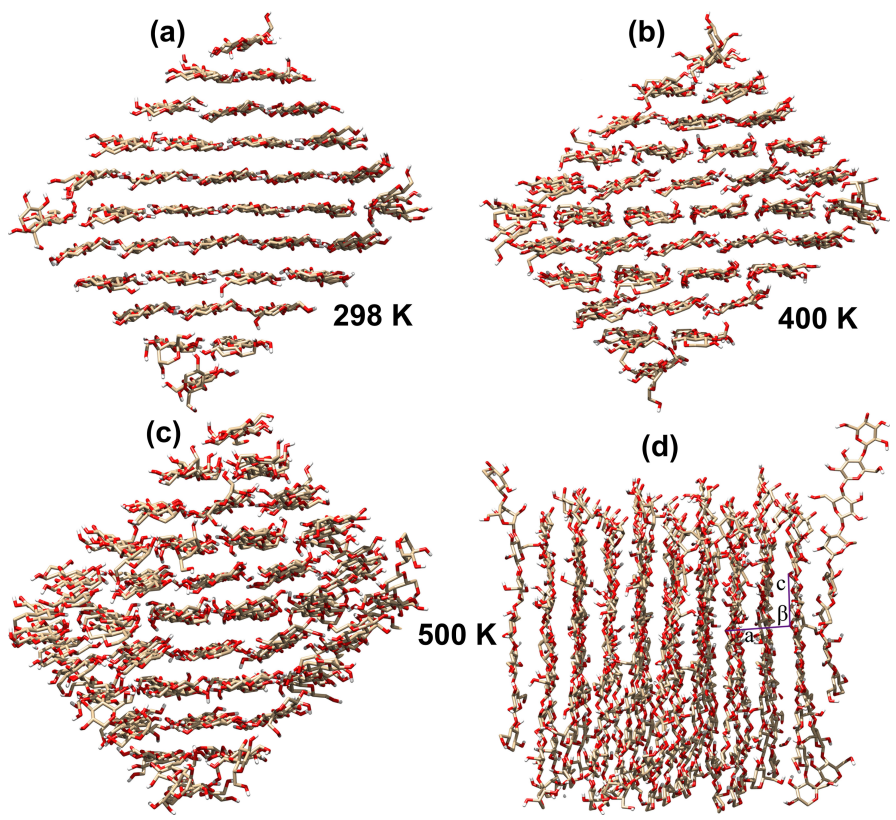

Figure 5: (a) Twisting effect on the structure of temperature $298 \mathrm{~K}$, (b) twisting effect on the structure of temperature $400 \mathrm{~K}$, and (c) twisting effect on the structure of temperature $500 \mathrm{~K}$, (d) chain sliding effect on the structure of temperature $500 \mathrm{~K}$.

number of twisting chains is mounting progressively with the temperature increasing from $350 \mathrm{~K}$ to $450 \mathrm{~K}$. This phenomena was also observed in experiments (HANLEY et al. 1997) and the previous MD simulations (Paavilainen et al. 2011). The twisting in structure results in an increment of the unit cell angle $\beta$ after 450 $\mathrm{K}$ as shown in Fig. 4. At $500 \mathrm{~K}$, two cellulose chains in adjacent sheets were further away from each other compared to that at lower temperature, suggesting that chains were separated from each other along the $c$ axis (Fig. 5d). This separation contributes to about 1.1\% increasing of unit cell angle $\beta$ compared to that at $400 \mathrm{~K}$ (Table 1). The change in $\boldsymbol{a}$ parameter is consist of two factors: the expanded distance between different sheets and the slippage along the molecule chains. If the distance was not enlarged between different sheets, a slight change in unit cell angle $\beta$ should cause only $0.04 \%$ increase of unit cell parameter $\boldsymbol{a}$ mathematically with the sine function. However, about $4 \%$ increase of parameter $\boldsymbol{a}$ was found in our study. Therefore, the increase of $\boldsymbol{a}$ parameter found in our study is mainly derived from the increase of expanded distance between different sheets.

We also analyzed the conformation of molecule chains in order to characterize the thermal response of the cellulose I $\beta$. The population of $\boldsymbol{g t}, \mathbf{t g}$ and $\mathbf{g g}$ conformations at different temperature can be estimated by the distribution of the torsion angle $\omega$ as shown in Fig. 6 . Without translational symmetry along the chain direction in the initial cellulose $\mathrm{I} \beta$ structure, the end residues of every chain were unable to be used in estimating the distribution of hydroxymethyl groups. At $298 \mathrm{~K}$, most of hydroxymethyls are in $\mathbf{t g}$ conformation, and the population of $\boldsymbol{g} \boldsymbol{g}$ and $\boldsymbol{g t}$ conformations are minority. This is in agreement with the X-ray diffraction data (Nishiyama et al. 2002).

A drastic change emerged at $350 \mathrm{~K}$ where about half of the hydroxymethyl groups convert from tg to the $\boldsymbol{g} \boldsymbol{t}$ or $\mathbf{g} \boldsymbol{g}$ conformation. In addition, the population of $\boldsymbol{g} \boldsymbol{g}$ conformation surpass that of $\boldsymbol{g} \boldsymbol{t}$ conformation. The gt and gg conformations represent rotated and non-rotated backbone chains respectively, according to the previous work (Zhang et al. 2011). Therefore, the observed twisting of cellulose chains can be attributed to bond rotations in order to adopt $\boldsymbol{g t}$ conformation. The $\boldsymbol{g} \boldsymbol{t}$ conformation became more populated when the temperature ascending from $400 \mathrm{~K}$ to $550 \mathrm{~K}$, while the population of $\mathbf{g g}$ conformation slightly decline. This result agrees with the work of Bergenstråhle (Bergenstrahle et al. 2007) that the population of $\boldsymbol{g t}$ conformation exceeds that of $\mathbf{g g}$ conformation at $550 \mathrm{~K}$, whereas original $\boldsymbol{t g}$ conformation become negligible. It was reported by former works that bond rotations often occur near the transition temperature (Bergenstrahle et al. 2007; Zhang et al. 2011). Although larger quantities of twisted chain along the $\boldsymbol{c}$ axis were observed in our 
study compared to others results (Bergenstrahle et al.2007; Zhang et al. 2011); however, this character agrees with experimental data that the $\mathbf{C 6}$ atom is more impressionable at high temperature compared to that at room temperature (Watanabe et al. 2006b).

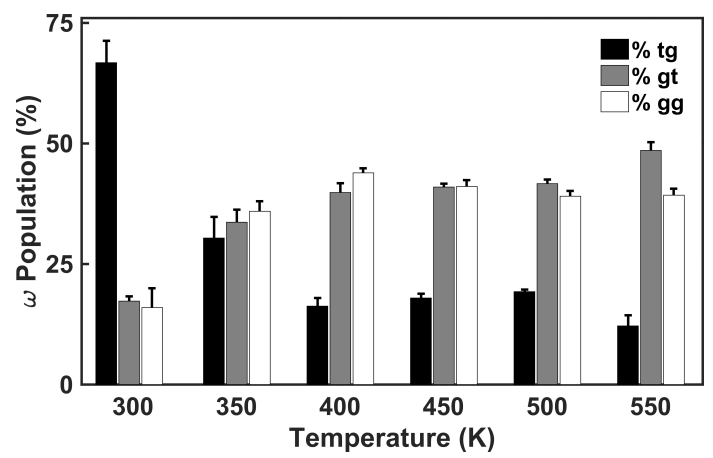

Figure 6: Populations of the three staggered conformations (tg, $\boldsymbol{g t}$ and $\boldsymbol{g g})$ of the torsion angle $\omega\left(\mathrm{O}_{5}-\mathrm{C}_{5}-\mathrm{C}_{6}-\mathrm{O}_{6}\right)$ at different temperature.

\subsection{Hydrogen Bonds}

The change in the orientation of the hydroxymethyl groups and glycosidic bond may lead to a different hydrogen bond network between cellulose molecule chains. Fig. 7 demonstrates the characteristics of hydrogen bonds in interior area that still maintain crystalline structure. The hydrogen bond cutoff was set as a bond length of $3.0 \AA$ and a bond angle of $150^{\circ}$. According to the studies of Nishiyama and others (Nishiyama et al. 2008; Nishiyama et al. 2002; Zhang et al. 2011), the hydrogen bonds in cellulose crystal structure can be classified into three types: intrachain, interchain and intersheet hydrogen bonds. The hydrogen bonds 03H03... 05 and $02-\mathrm{HO} 2 . .06$ are the major types of intrachain and the hydrogen bond 06-HO6...03' is the major type of interchain in the native cellulose I $\beta$ (Fig. 7a). The intrachain hydrogen bond 03-H03...05 is constructed by hydroxyl group $3 \mathrm{OH}$ at the $\mathrm{C} 3$ position with the $\mathrm{O} 5$ atom in the adjacent ring. The intrachain hydrogen bond $\mathrm{O} 2-\mathrm{HO} 2 . . .06$ is formed by group $2 \mathrm{OH}$ at the $\mathrm{C} 2$ with the $\mathrm{O} 6$ atom in the adjacent ring, while the hydroxyl group $6 \mathrm{OH}$ at the $\mathrm{C} 6$ position and the $\mathrm{O} 3$ atom in the neighboring chain induce interchain hydrogen bond formation $06-\mathrm{HO} 6 . . .03^{\prime}$.

At $298 \mathrm{~K}$, the intrachain hydrogen bond 03-H03...05, 02-HO2...06 and interchain hydrogen bond 06HO6... O $3^{\prime}$ are characterized. This result is consistent with the dominant hydrogen bond network determined by the experiments and simulations (Hayakawa et al. 2017; Nishiyama et al. 2008; Nishiyama et al. 2002; Zhang et al. 2011). The population of intrachain hydrogen bond O2-HO2...06 is $91 \%$ and interchain hydrogen bond $06-H 06 . . .03$ ' is $82 \%$ instead of $100 \%$ (Nishiyama et al. 2002; Zhang et al. 2011). This deviation may because of disorder in cellulose I $\beta$ structure (Nishiyama et al. 2002; Nishiyama et al. 2003). The simulation result is in agreement with previous findings that the hydrogen bond network in cellulose $\mathrm{I} \beta$ is stable at room temperature (Mazeau 2005; Nishiyama et al. 2008). The intersheet hydrogen bond O-HO... O was not observed at temperature of $298 \mathrm{~K}$ (Fig. 7b), which is corresponding to the experimental results (Nishiyama et al. 2002). Above results indicate that the hydrogen bonds are critical to stability of intrachain and interchain but not to intersheet.

We also analysis the alternation of hydrogen bond network pattern under various temperatures in this study. In general, the populations of the intrachain hydrogen bonds 03-HO3...05, 02-HO2...06 and interchain hydrogen bond 06-H06...03' decrease with the temperature rising (Fig. 7b). Combing with the structural alternation of cellulose above, it is obvious that hydrogen bond network contributed to the chain rearrangement under different temperatures. 


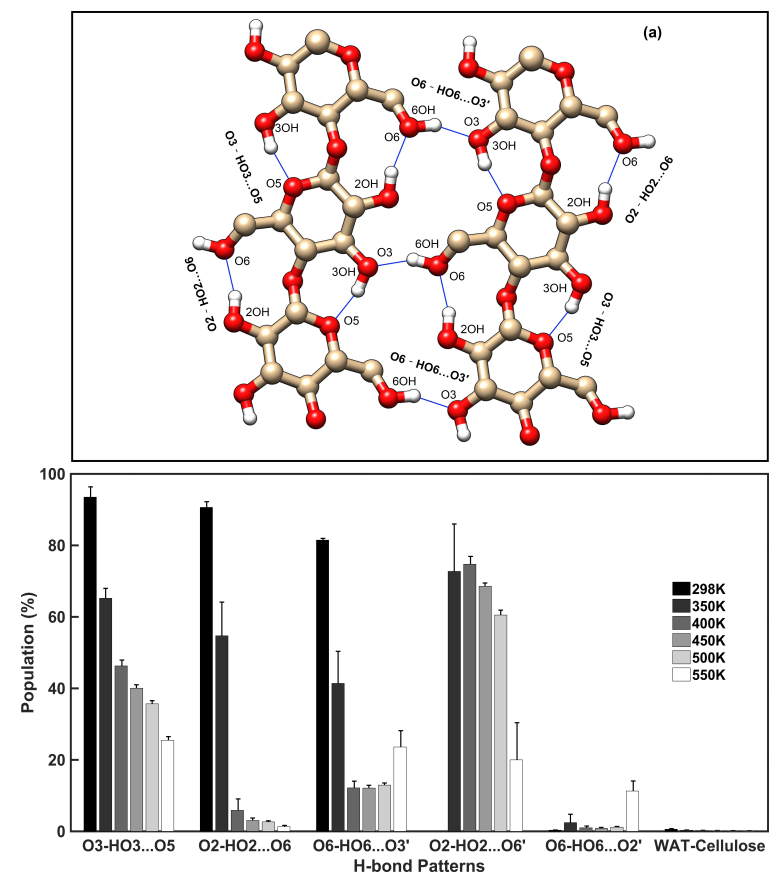

Figure 7: The character of $\mathrm{H}$-bond of cellulose $\mathrm{I} \beta$ at different temperature. (a) The blue lines indicate the proposed cooperative networks of hydrogen bonds. (b) Population of maximum possible intrachain and interchain hydrogen bonds.

When the system was heated from $298 \mathrm{~K}$ to $350 \mathrm{~K}$, the population of the hydrogen bond 03-HO3...05 decreased by $28 \%$ and the hydrogen bond $02-\mathrm{H} 2 . . .06$ decreased by $36 \%$. Although about half of the hydroxymethyl groups convert from tg to the gt or gg conformations at $350 \mathrm{~K}$, most of them occurred at the cellulose/water interface. In the addition, the hydrogen bond was estimated by crystal internal 16 different unit cells in the system. This leads to about $60 \%$ of the intramolecular hydrogen bond of $\mathrm{C} 2 \mathrm{OH}-06$ still remained. These results are partially in agreement with the experimental data provided by studies using IR spectroscopy, in which the alternation of intrachain hydrogen bond 03-H03...05 and 02-HO2...06 was observed within the temperature range of 303-473 K (Watanabe et al. 2006a; Watanabe et al. 2006b). This is also comparable with previous MD simulations, in which a $40 \%$ decreasing of the interchain hydrogen bond 06HO6... O3' was observed when the temperature was raised from $298 \mathrm{~K}$ to $350 \mathrm{~K}$ (Zhang et al. 2011). In addition, a new type of interchain hydrogen bond $02-\mathrm{HO}^{2} . .06^{\prime}$ was formed with a population of $72 \%$ at 350 $\mathrm{K}$. This can be attributed to the conformational changes of the hydroxymethyl groups in interior area. The change of populations of intrachain and interchain hydrogen bonds indicates that hydrogen bonding pattern at $298 \mathrm{~K}$ translated to another one when the system was heated to $350 \mathrm{~K}$.

On the other hand, at $500 \mathrm{~K}$, the population of the hydrogen bond $03-\mathrm{HO} 3 \ldots .05$ decreased to about $36 \%$ and the hydrogen bond $02-\mathrm{HO} 2 . .06$ has only about $3 \%$ left. The interchain hydrogen bond $06 \mathrm{HO} 6 \ldots \mathrm{O}^{\prime}$ and O2-HO2...06' decreased to $13 \%$ and $61 \%$ respectively, which are in line with the previous MD simulations (Bergenstrahle et al. 2007; Zhang et al. 2011). The population of 02-HO2...06' hydrogen bond is $13 \%$ less at $500 \mathrm{~K}$ compare that at $400 \mathrm{~K}$. These results imply the changing of structural pattern of cellulose crystals. Therefore, it can be concluded that the structure of cellulose I $\beta$ at high temperature introduced a less ordered nature.

Minor difference in hydrogen bonds network in response to the temperature change is demonstrated as bond distance distribution of the hydrogen bonds as shown in Fig. 8. The most probable hydrogen acceptor distance of $03-\mathrm{HO} 3 . .05$ is $2.76 \AA$ at $298 \mathrm{~K}$, which is slightly shorter than that under other temperatures. The population of 03-HO3...05 hydrogen bond is $16.1 \%$ at $298 \mathrm{~K}$ (Fig. 8a), which is slightly larger than that under other temperatures. These phenomenon of 03-HO3... 05 hydrogen bond are in agreement with findings of previous MD simulations (Bergenstrahle et al. 2007). The ranges of distance from $2.5 \AA$ to $3.1 \AA$ (Fig. 8b) showing that the intrachain hydrogen bond $02-\mathrm{HO} 2 . . .06$ at lower temperature range $(298 \mathrm{~K}$ and $350 \mathrm{~K})$ are 

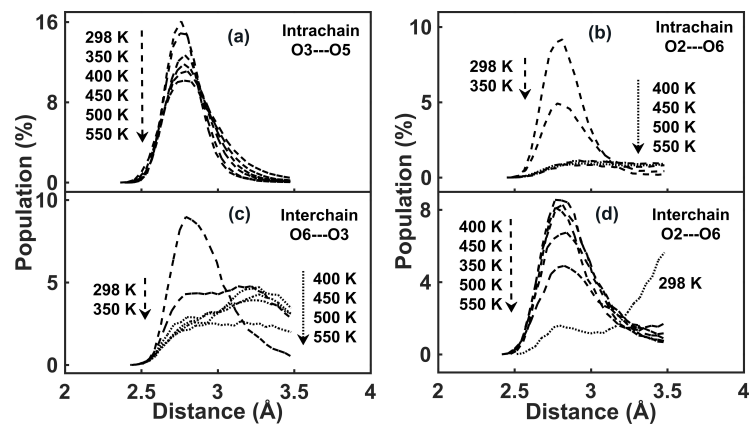

Figure 8: Population of intrachain and interchain bonds distance distributions. (a) Distance distributions of the intrachain 0305, (b) the distance distributions of the intrachain 02-06, (c) distance distributions of the interchain 06-03' and (d) distance distributions of the interchain 02-06'.

stronger than that under higher temperature ( $400 \mathrm{~K}$ to $550 \mathrm{~K}$ ). Likewise, the intrachain hydrogen bonds 03HO3... O5 (Fig. 8a) and O2-HO2... O6 (Fig. 8b) are stronger than the interchain hydrogen bonds 06HO6...03' (Fig. 8c) and 02-HO2... O6' (Fig. 8d) at lower temperature range. These results are consistent with the previous works (Bergenstrahle et al. 2007; Zhang et al. 2011). The most probable hydrogen acceptor distance of the newly formed O2-HO2... O6' (Fig. 8d) hydrogen bond is about $2.76 \AA$ at $400 \mathrm{~K}$, shorter than that (2.84 $\mathrm{A}$ ) at $500 \mathrm{~K}$ and the corresponding population of $02-\mathrm{HO} 2 . . .06^{\prime}$ hydrogen bond decreases from $400 \mathrm{~K}$ to $500 \mathrm{~K}$. These can be explained by the fact that a few hydroxymethyl groups transform to the gt conformation from gg conformation, contributing to the fact of weakening the hydrogen bond strength.

\section{Conclusions}

In a summary, thermal response of cellulose $\mathrm{I} \beta$ was reported by molecular dynamics simulations with the GLYCAM06 force field in the present paper. All simulations reached equilibrium with the RMSD and total energy used as the indicator of equilibrated system.

With less than 3.9\% deviations of density and unit cell parameters between the data measured in previous experiments and that in our simulation performed at $298 \mathrm{~K}$, the computational methods and selection of force field in our study was proved with validity for further analysis of the structure. In addition, the hydrogen bonding pattern calculated at temperature $298 \mathrm{~K}$ was in sufficient accordance with that measured experimentally, herein as another support of this computational simulation.

A determined structure transition temperature (about $450 \mathrm{~K}$ ) was consistent with the experimental data, meanwhile, another transition temperature around $350 \mathrm{~K}$ was detected in our simulation. Three structures at low, intermediate and high temperature was identified and demonstrated individually in this work. In the intermediate temperature structure, twisting emerged in a few chains along the chain direction. We also observed the sudden changing of density and unit cell parameters $\boldsymbol{a}, \boldsymbol{b}, \alpha$ and $\gamma$ at this intermediate temperature. In addition, the conformational changes of hydroxymethyl groups from $\boldsymbol{t g}$ to $\mathbf{g t}$ and $\mathbf{g g}$ were observed when raising the system temperature. Note that the $\mathbf{g g}$ conformation was dominant into conformational alternation. This also contributes to the transitions of the hydrogen bonding pattern under various temperature. When the temperature was further increased to $400 \mathrm{~K}$, majority of the hydrogen bonds $02-\mathrm{HO} 2 . .06$ and 06-HO6...03' were disrupted and substituted by interchain hydrogen bonds $02-\mathrm{HO} 2 \ldots 06^{\prime}$. The structural responses of cellulose $\mathrm{I} \beta$ at higher temperature were illustrated as consequences of chain sliding along $\mathrm{c}$ axis, bond rotations, drastic changing of density and unit cell parameters $\boldsymbol{a}, \boldsymbol{b}$ and $\beta$, then the population of hydroxymethyl groups in $\mathbf{g t}$ and $\mathbf{g g}$ exchanged, therefore a conversion of hydrogen bonding pattern.

The structures under low and high temperature in our work were in reasonable agreement with the results obtained by the previous MD simulations and experiments. The discovery and illustration of three structures of cellulose $\mathrm{I} \beta$ that existed simultaneously under different temperature by molecular dynamic simulation will 
contribute to understanding the details of structural properties at molecular level. The simulated trajectories demonstrate details of thermal response of cellulose $\mathrm{I} \beta$ by providing the structures from atoms to hydrogen bond patterns, explaining the physical phenomenon that observed in experimental studies at a molecular level.

Acknowledgement: This work was supported by the Hubei Key Laboratory of Biomass Fibers and Eco-dyeing \& Finishing [grant numbers STRZ2018004]. We would like to thank the Biomolecular Physics and Modeling Group, Huazhong University of Science and Technology for computing resources.

\section{References}

[1] Atalla RH, Vanderhart DL (1984) Native cellulose: a composite of two distinct crystalline forms Science 223:283-285

[2] Berendsen H, van Postma JPM, van Gunsteren W, DiNola AD, Haak JR (1984) Molecular-Dynamics with Coupling to An External Bath vol 81. doi:10.1063/1.448118

[3] Bergenstrahle M, Berglund LA, Mazeau K (2007) Thermal response in crystalline lbeta cellulose: a molecular dynamics study J Phys Chem B 111:9138-9145 doi:10.1021/jp072258i

[4] Cai L, Liu Y, Liang H (2012) Impact of hydrogen bonding on inclusion layer of urea to cellulose: Study of molecular dynamics simulation Polymer 53:1124-1130

[5] Chen L, Qin Y, Wang X, Zhao X, Wang F (2011) Plasticizing while toughening and reinforcing poly(propylene carbonate) using low molecular weight urethane: Role of hydrogen-bonding interaction Polymer 52:4873-4880

[6] Chen P, Ogawa Y, Nishiyama Y, Ismail AE, Mazeau K (2018) I $\alpha$ to I $\beta$ mechano-conversion and amorphization in native cellulose simulated by crystal bending Cellulose 25:4345-4355

[7] D.A. Case et al. (2012) AMBER 12 University of California, San Francisco

[8] DeMarco ML, Woods RJ (2009) Atomic-resolution conformational analysis of the GM3 ganglioside in a lipid bilayer and its implications for ganglioside-protein recognition at membrane surfaces Glycobiology 19:344-355

[9] DeMarco ML, Woods RJ, Prestegard JH, Tian F (2010) Presentation of membrane-anchored glycosphingolipids determined from molecular dynamics simulations and NMR paramagnetic relaxation rate enhancement Journal of the American Chemical Society 132:1334-1338

[10] Ding S-Y, Himmel ME (2006) The Maize Primary Cell Wall Microfibril: A New Model Derived from Direct Visualization J Agric Food Chem 54:597-606 doi:10.1021/jf051851z

[11] Gardner K, Blackwell J (1974) The structure of native cellulose Biopolymers 13:1975-2001

[12] Gomes TC, Skaf MS (2012) Cellulose-Builder: A toolkit for building crystalline structures of cellulose Journal of computational chemistry 33:1338-1346

[13] HANLEY SJ, REVOL J-F, GODBOUT L, GRAY DG (1997) Atomic force microscopy and transmission electron microscopy of cellulose from Micrasterias denticulata; evidence for a chiral helical microfibril twist Cellulose 4:209 doi:10.1023/a:1018483722417

[14] Hayakawa D, Nishiyama Y, Mazeau K, Ueda K (2017) Evaluation of hydrogen bond networks in cellulose I $\beta$ and II crystals using density functional theory and Car-Parrinello molecular dynamics Carbohydr Res 449:103-113

[15] Heiner AP, Kuutti L, Teleman O (1998) Comparison of the interface between water and four surfaces of native crystalline cellulose by molecular dynamics simulations Carbohydr Res 306:205-220

[16] Heiner AP, Sugiyama J, Teleman $O$ (1995) Crystalline cellulose $I \alpha$ and I $\beta$ studied by molecular dynamics simulation Carbohydr Res 273:207-223

[17] Horii F, Yamamoto H, Kitamaru R, Tanahashi M, Higuchi T (1987) Transformation of native cellulose crystals induced by saturated steam at high temperatures Macromolecules 20:2946-2949

[18] Huang MR, Li XG (1998) Thermal degradation of cellulose and cellulose esters J Appl Polym Sci 68:293-304

[19] Jorgensen WL, Chandrasekhar J, Madura JD, Impey R, Klein ML (1983) Comparison of simple potential functions for simulating liquid water Journal of Chemical Physics 79:926-935

[20] Kirschner KN, Yongye AB, Tschampel SM, González-Outeiriño J, Daniels CR, Foley BL, Woods RJ (2008) GLYCAM06: a generalizable biomolecular force field. Carbohydrates Journal of computational chemistry 29:622-655

[21] Liu H, Sale KL, Simmons BA, Singh S (2011) Molecular dynamics study of polysaccharides in binary solvent mixtures of an ionic liquid and water J Phys Chem B 115:10251-10258

[22] Makhatadze GI, Privalov PL (1992) Protein interactions with urea and guanidinium chloride: a calorimetric study Journal of molecular biology 226:491-505

[23] Mazeau K (2005) Structural micro-heterogeneities of crystalline I $\beta$-cellulose Cellulose 12:339-349

[24] Miyamoto H, Schnupf U, Crowley MF, Brady JW (2016) Comparison of the simulations of cellulosic crystals with three carbohydrate force fields Carbohydr Res 422:17-23 
[25] Miyamoto H, Yamane C, Ueda K (2013) Structural changes in the molecular sheets along (hk0) planes derived from cellulose I by molecular dynamics simulations Cellulose 20:1089-1098

[26] Miyamoto S, Kollman PA (1992) SETTLE: An analytical version of the SHAKE and RATTLE algorithm for rigid water models ] Comput Chem 13:952-962

[27] Nishiyama Y, Johnson GP, French AD, Forsyth VT, Langan P (2008) Neutron crystallography, molecular dynamics, and quantum mechanics studies of the nature of hydrogen bonding in cellulose Ibeta Biomacromolecules 9:3133-3140

[28] Nishiyama Y, Langan P, Chanzy H (2002) Crystal structure and hydrogen-bonding system in cellulose Ibeta from synchrotron $X$-ray and neutron fiber diffraction J Am Chem Soc 124:9074-9082

[29] Nishiyama Y, Sugiyama J, Chanzy H, Langan P (2003) Crystal Structure and Hydrogen Bonding System in Cellulose I $\alpha$ from Synchrotron X-ray and Neutron Fiber Diffraction Journal of the American Chemical Society 125:14300-14306

[30] Paavilainen S, Rog T, Vattulainen I (2011) Analysis of twisting of cellulose nanofibrils in atomistic molecular dynamics simulations J Phys Chem B 115:3747-3755 doi:10.1021/jp111459b

[31] Pace C (1986) Determination and analysis of urea and guanidine hydrochloride denaturation curves Methods in enzymology 131:266

[32] Rabideau BD, Agarwal A, Ismail AE (2013) Observed Mechanism for the Breakup of Small Bundles of Cellulose I $\alpha$ and I $\beta$ in Ionic Liquids from Molecular Dynamics Simulations J Phys Chem B 117:3469-3479

[33] Shiiba H, Hayashi S, Yui T (2013) Molecular dynamics study of carbohydrate binding module mutants of fungal cellobiohydrolases Carbohydr Res 374:96-102

[34] Sindhikara DJ, Kim S, Voter AF, Roitberg AE (2009) Bad Seeds Sprout Perilous Dynamics: Stochastic Thermostat Induced Trajectory Synchronization in Biomolecules Journal of Chemical Theory and Computation 5:1624-1631

[35] Spiwok V, Králová B, Tvaroška I (2010) Modelling of $\beta$-d-glucopyranose ring distortion in different force fields: a metadynamics study Carbohydr Res 345:530-537

[36] Stortz CA, Johnson GP, French AD, Csonka GI (2009) Comparison of different force fields for the study of disaccharides Carbohydr Res 344:2217-2228

[37] Sugiyama J, Vuong R, Chanzy H (1991) Electron diffraction study on the two crystalline phases occurring in native cellulose from an algal cell wall Macromolecules 24:4168-4175

[38] Swatloski RP, Spear SK, Holbrey JD, Rogers RD (2002) Dissolution of cellose with ionic liquids Journal of the American Chemical Society 124:4974-4975

[39] Tessier MB, DeMarco ML, Yongye AB, Woods RJ (2008) Extension of the GLYCAM06 biomolecular force field to lipids, lipid bilayers and glycolipids Molecular simulation 34:349-364

[40] Wada M (2002) Lateral thermal expansion of cellulose I $\beta$ and IIII polymorphs Journal of Polymer Science Part B: Polymer Physics 40:1095-1102

[41] Wada M, Hori R, Kim U-J, Sasaki S (2010) X-ray diffraction study on the thermal expansion behavior of cellulose I $\beta$ and its high-temperature phase Polymer Degradation and Stability 95:1330-1334

[42] Watanabe A, Morita S, Ozaki Y (2006a) Study on temperature-dependent changes in hydrogen bonds in cellulose $I \beta$ by infrared spectroscopy with perturbation-correlation moving-window two-dimensional correlation spectroscopy Biomacromolecules 7:3164-3170

[43] Watanabe A, Morita S, Ozaki Y (2006b) Temperature-dependent structural changes in hydrogen bonds in microcrystalline cellulose studied by infrared and near-infrared spectroscopy with perturbation-correlation moving-window twodimensional correlation analysis Appl Spectrosc 60:611-618

[44] Wyman CE (2007) What is (and is not) vital to advancing cellulosic ethanol Trends Biotechnol 25:153-157

[45] Yamane C, Miyamoto H, Hayakawa D, Ueda K (2013) Folded-chain structure of cellulose II suggested by molecular dynamics simulation Carbohydr Res 379:30-37

[46] Yui T, Nishimura S, Akiba S, Hayashi S (2006) Swelling behavior of the cellulose I $\beta$ crystal models by molecular dynamics Carbohydr Res 341:2521-2530

[47] Zhang Q, Bulone V, Ågren H, Tu Y (2011) A molecular dynamics study of the thermal response of crystalline cellulose $1 \beta$ Cellulose 18:207-221

[48] Zou Q, Bennion BJ, Daggett V, Murphy KP (2002) The molecular mechanism of stabilization of proteins by TMAO and its ability to counteract the effects of urea Journal of the American Chemical Society 124:1192-1202 